\title{
Utilization of Acupuncture Therapy among Pediatric Oncology Patients at a Tertiary Care Pediatric Hospital
}

\author{
Ralston-Wilson $\mathbf{J}^{1,3,6 *}$, Tseng $A^{1}$, Oberg $E^{2}$, Sasagawa $M^{1}$, Doorenbos $A Z^{4,5}$ and Kundu $A^{2}$ \\ ${ }^{1}$ Bastyr University, Kenmore, WA, USA \\ ${ }^{2}$ Clinical Services and Center for Health Policy and Leadership, Bastyr University, San Diego, CA, USA \\ ${ }^{3}$ Department of Anesthesiology and Pain Medicine, Seattle Children's Hospital, Seattle, WA, USA \\ ${ }^{4}$ Department of Anesthesiology and Pain Medicine School of Medicine, University of Washington, Seattle, WA, USA \\ ${ }^{5}$ Department of Biobehavioral Nursing and Health Systems, University of Washington, Seattle, WA, USA \\ ${ }^{6}$ Complementary and Integrative Medicine Program, Seattle Children's Hospital, Seattle, WA, USA
}

\begin{abstract}
Background: Acupuncture is infrequently studied in the pediatric oncology population. However its potential as a complementary cancer care modality in the reduction of treatment-related side effects and improvements in quality of life has been demonstrated in adults. Our study aims to characterize the patients and utilization of acupuncture therapy among the pediatric population.
\end{abstract}

Methods: A retrospective analysis of medical records for patients between the ages of 0-18 years, undergoing treatment for an oncological diagnosis and had received acupuncture therapy between January 2004 and September 2012 was conducted.

Results: A total of 133 patients, comprising $3.2 \%$ of our pediatric oncology population, utilized acupuncture therapy. A cumulative of 523 acupuncture treatments was provided. Treatments were tolerated well by patients, perceived as beneficial, and without any serious adverse events. Acupuncture treatments addressed a wide variety of patients' complaints and cancer treatment-related side effects.

Conclusions: Acupuncture use among the pediatric oncology population is relatively low; however, our findings demonstrate its potential as a complementary cancer care modality in clinical practice for this unique population in the future.

Keywords: Acupuncture; Pediatric; Oncology

Abbreviations: CAM: Complementary and Alternative Medicine; TCM: Traditional Chinese Medicine; NRS: Numeric Rating Scale; FLACC: Face, Legs, Activity, Cry, Consolability Scale; CNS: Central Nervous System; MDS: Myelodysplastic Syndrome

\section{Introduction}

Acupuncture therapy has increasingly been utilized as a complementary treatment modality both for a wide variety of cancerrelated conditions and for side effects related to cancer treatments [111]. Adult cancer patients have experienced an improvement in the quality of life as a result of the positive effects of acupuncture when it is included as an adjunctive therapy for management of a multitude of complaints [1-9]. While the use of acupuncture has become more common in cancer care, there remains a relative paucity of evidencebased research providing safety and efficacy data among oncology patients, thus limiting the extent to which patients and providers feel confident in pursuing or recommending the use of acupuncture in oncological care [3-9]. This may be especially true in the pediatric population where acupuncture research is even more sparce [12-18]. The application of acupuncture therapy among the pediatric oncology population remains largely unexplored [12-15]. Despite the reassuring positive results attributable to acupuncture therapy in the adult population, it is important to consider the uniqueness and differences in the pediatric population prior to the anticipation of similar results in children. Therefore, we present our findings on the utilization trends, referral patterns, adverse events, and subjective experience of pediatric oncology patients who underwent acupuncture therapy at a tertiary care pediatric hospital in the Pacific Northwest United States.

\section{Materials and Methods}

\section{Setting}

After obtaining approval from the institutional review boards of the pediatric hospital and collaborating university, we conducted a retrospective chart review and analysis of pediatric cancer patients that received acupuncture therapy between the years of 2004-2012 at Seattle Children's Hospital.

Seattle Children's Hospital is a tertiary care pediatric hospital serving a diverse population drawn from a five-state region. Acupuncture therapy was introduced at our institution in the latter part of 2004 with a single physician acupuncturist initially providing this therapy. However, the number of acupuncture providers has gradually increased to currently include 3 anesthesiologists who have also trained as physician acupuncturists and 1 licensed acupuncturist who is employed part-time. Acupuncture therapy is not a standard of care at Seattle Children's Hospital for all patients, however it does

*Corresponding author: Jaime Ralston-Wilson, Department of Anesthesiology and Pain Medicine, M/S MB.11.500.3 Anesthesia Administration, Seattle Children's Hospital, 4800 Sand Point Way NE, Seattle, WA 98105, USA, Tel: (206) 987-2704; Fax: (206) 987-3935; E-mail: jaime.ralstonwilson@seattlechildrens.org

Received June 02, 2013; Accepted July 08, 2013; Published July 10, 2013

Citation: Ralston-Wilson J, Tseng A, Oberg E, Sasagawa M, Doorenbos AZ, et al. (2013) Utilization of Acupuncture Therapy among Pediatric Oncology Patients at a Tertiary Care Pediatric Hospital. Altern Integ Med 2: 129. doi:10.4172/2327 5162.1000129

Copyright: ( 2013 Ralston-Wilson J, et al. This is an open-access article distributed under the terms of the Creative Commons Attribution License, which permits unrestricted use, distribution, and reproduction in any medium, provided the original author and source are credited. 
not preclude standard of care and is rather provided in addition to the standard therapies. Provision of acupuncture therapy depends on the availability of an acupuncturist and requires a consultation request from the patient's clinical provider.

In this study, we reviewed transactional data from the medical records of pediatric cancer patients who were treated with acupuncture therapy at Seattle Children's Hospital during the years 2004-2012. We systematically characterized the utilization of acupuncture therapy, as well as the patients with oncological diagnoses who pursued acupuncture therapy as a treatment modality during this time period. Further, we also conducted a sub-analysis of the acupuncture treatments that were received by these patients.

\section{Sample}

The number of cases anticipated for review was determined using a statistical confidence level of $95 \%$ and a margin of error of $5 \%$ using Raosoft ${ }^{\circledR}$ sample size calculator (http://www.raosoft.com/samplesize. html) assuming the response distribution of 0.5 , which is the most conservative estimate [19]. Based on these calculations, the number of patients was not to exceed 384 cases. If the number of eligible subjects was to exceed this number, patient charts would have been selected randomly by using a random generator or other randomized selection method. It was not necessary to use a random selection method as our population was smaller than the anticipated maximum.

\section{Inclusion criteria}

Children between the ages of $0-18$ years, with an oncological diagnosis, receiving cancer treatments and who had undergone acupuncture therapy between January 1, 2004 and September 30, 2012 were included in our study.

\section{Data collection}

Data was obtained through the use of hospital Health Information Management System that includes the scheduling and billing software searchable by specific fields. Our search included information based on age, date of service, acupuncture procedure codes, and oncological diagnosis codes. We also collected data regarding patients' birth year, gender, mortality status, zip code, state of residence, race, ethnicity, acupuncture service date range, diagnosis codes for acupuncture therapy, and total number of acupuncture treatments received.

Additional data collected on patients meeting the inclusion criteria was upon review of patients' electronic, paper, and microfilm records. Further oncological data included cancer type and disease characteristics. Additionally, detailed information pertaining to specific acupuncture treatments was also collected including; age at time of treatment, inpatient or outpatient status, specialty of the referring clinician requesting acupuncture consult, reason for referral, chief complaints treated, tongue, pulse, abdominal, and traditional Chinese medicine (TCM) diagnosis assigned to the patient by the acupuncture provider, acupuncture points used during treatment, total number of acupuncture needles used during a treatment, modality used for acupuncture point stimulation, length of acupuncture treatment or needle retention time, pain scale rating using numeric rating scale (NRS) or the face, legs, activity, cry, consolability (FLACC) scale, response to acupuncture therapy as reported by the patient, family, and/ or practitioner, and acupuncture-related adverse events from either the current or past treatment.

Adverse events related to acupuncture therapy were categorized as present or absent. Specific adverse events included in this review were; allergic reaction due to metal needles, bleeding requiring digital pressure or further intervention, bruising or hematoma, burn due to moxibustion, discomfort during or after treatment, dizziness, fainting, fatigue, headache, nausea/vomiting, infection, localized skin irritation, nerve injury or damage, pain at puncture site during insertion, removal, or after treatment, pneumothorax, organ puncture or damage, and other including central nervous system (CNS) injuries.

\section{Results}

During the time period January 2004-September 2012, a total of 4169 patients were identified as carrying an oncological diagnosis and 455 patients were identified as patients that received acupuncture therapy during this time period at our institution. Based on our inclusion criteria, 133 patients met the requirements as both having an oncological diagnosis and received acupuncture therapy during the study time period (Figure 1).

\section{Patient demographics}

The demographic information (Table 1 ) includes a nearly matched gender distribution with $65(48.9 \%)$ male and 68 (51.1\%) female. The mean age at the first acupuncture therapy appointment was 12.3 years, with a median age of 9 years, and mode of 18 years. The majority, $106(79.9 \%)$ patients were residents of Washington State. Eighty-one $(60.9 \%)$ patients were Caucasian and as of the time of our initial data collection in February 2013, 92 (69.2\%) of the patients were still alive.

\section{Oncological diagnoses}

A majority of patients (91 or 68.4\%) were diagnosed with hematological cancers. Of the hematological cancer patients, leukemia was the most common diagnosis ( 66 or $49.6 \%$ ), followed by lymphoma (13 or $9.8 \%$ ) and myelodysplastic syndrome (MDS) (7 or 5.3\%). Of the $60(45 \%)$ pediatric patients with solid tumors, diagnoses included brain/CNS tumors (15 or 11.3\%); Ewing's sarcoma ( 9 or $6.8 \%$ ); neuroblastoma (8 or $6.0 \%)$; osteosarcoma (5 or $3.8 \%$ ); other sarcomas (5 or $3.8 \%$ ); rhabdomyosarcoma (3 or $2.3 \%$ ); hepatoblastoma (3 or $2.3 \%)$; Wilms' tumor/nephroblastoma (2 or $1.5 \%$ ); renal cell carcinoma (1 or $0.8 \%$ ); and neurofibromatosis (5 or 3.8\%); comprised the rest of the population. Nine (6.8\%) patients were categorized as having "other" oncological diagnoses. Of the seven patients diagnosed with MDS, five of them had subsequently developed leukemia. Eighteen (13.5\%) patients had multiple oncological diagnoses, fourteen of which carried a leukemia diagnosis.

\section{Acupuncture therapy utilization}

The total number of acupuncture treatments performed during

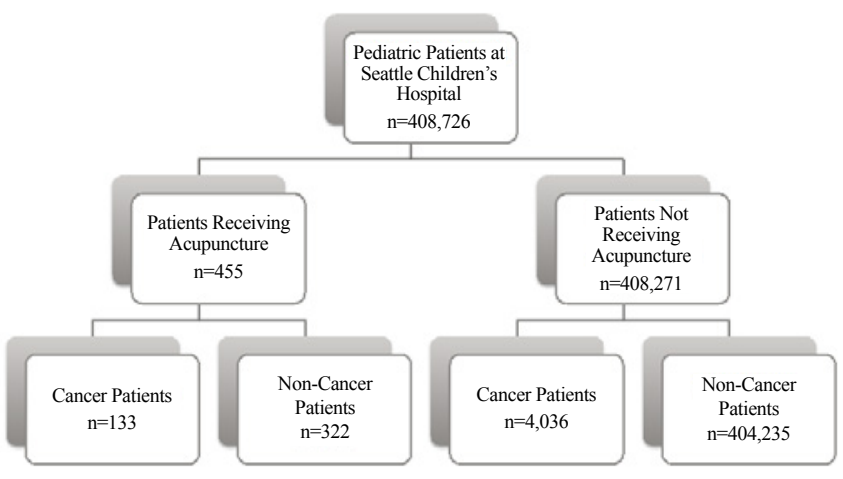

Figure 1: Characterization of acupuncture Use. 


\begin{tabular}{|l|c|c|}
\hline & $\mathbf{n}$ & $\%$ \\
\hline Gender & & \\
\hline Male & 65 & 48.9 \\
\hline Female & 68 & 51.1 \\
\hline Age at First Visit & & \\
\hline $0-3$ & 12 & 9 \\
\hline $4-9$ & 22 & 16.5 \\
\hline $10-12$ & 20 & 15 \\
\hline $13-18$ & 79 & 59.4 \\
\hline State of Residence & & \\
\hline WA & 106 & 79.7 \\
\hline MT & 6 & 4.5 \\
\hline AK & 5 & 3.8 \\
\hline CA & 5 & 3.8 \\
\hline Other & 11 & 8.4 \\
\hline Race/Ethnicity & 81 & \\
\hline Caucasian & 0 & 60.9 \\
\hline Black or African American & 14 & 10.5 \\
\hline Hispanic, Latino, or Spanish origin & 11 & 8.3 \\
\hline Asian & 1 & 0.8 \\
\hline Native Hawaiian/Other Pacific Islander & 1 & 0.8 \\
\hline American Indian/Alaska Native & 1 & 0.8 \\
\hline Mixed & 13 & 9.8 \\
\hline Other & 11 & 8.3 \\
\hline Unknown & 92 & 69.2 \\
\hline Mortality Status & 41 & 30.8 \\
\hline Alive & & \\
\hline Deceased & & \\
\hline & & \\
\hline
\end{tabular}

Table 1: Patient demographics.

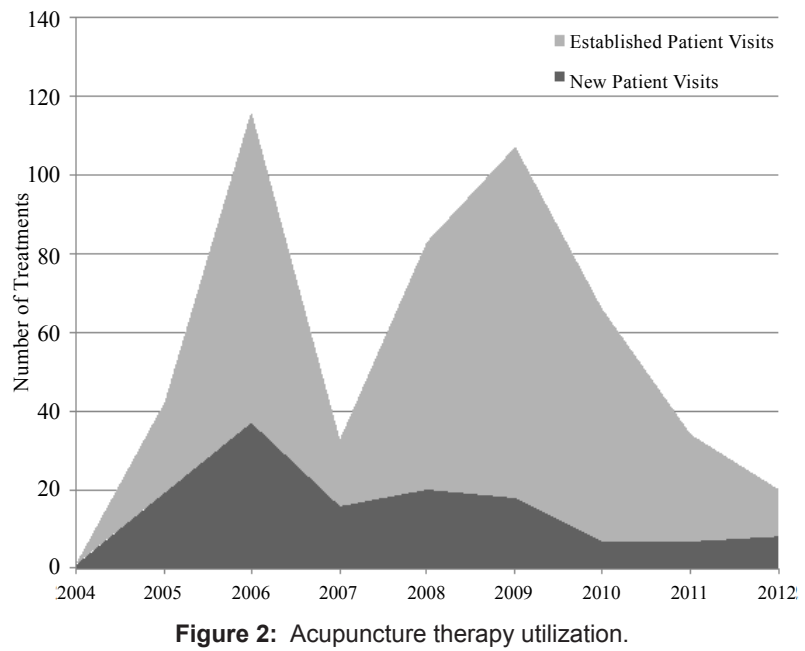

this time period was 523. Complete treatment data was available for approximately $85 \%$ of treatments. At times when data was not available, details for these treatments were either not documented by the practitioner, not present in the records that were available for review, or had very limited treatment specific data recorded.

Acupuncture treatments in the pediatric oncology population, were provided by 4 different providers; three physician acupuncturists and one licensed acupuncturist. A slightly larger number of acupuncture treatments were performed in the outpatient setting (257 or $49.6 \%$ ), while inpatient treatments accounted for 205 (39.6\%) of the total acupuncture visits. Outpatient or inpatient status was not clearly documented for $61(11.7 \%)$ acupuncture treatments received. The average number of acupuncture treatments received among the study population was 3.9. Although the number of acupuncture treatments ranged from 1 to 98 , the majority $(85.0 \%)$ of patients received between 1-5 treatments.

Trends in utilization of acupuncture therapy, amongst both new and established oncology patients, showed an expected initial increase at the introduction of the acupuncture service. The number of new patient acupuncture visits among oncology patients subsequently declined in some of the following years, while the established oncology patient visits varied with two prominent peaks in utilization (Figure 2). This fluctuation was partly due to variation in the number and availability of acupuncture providers, as well as high utilization among several of the patients with the greatest acupuncture usage. Dates of service were not available for $21(5.4 \%)$ acupuncture treatments received.

\section{Acupuncture referrals}

During the study period, the two largest documented sources for referrals were either from the hematology-oncology department (64 or $35.0 \%$ ) or from a parent or family member (60 or $32.8 \%$ ). Other prominent referral sources were the pain medicine team, bone marrow transplant service, and self/patient. Documentation of referral sources was only available for 86 patients (64.7\%) and 131 treatments (25\%), although at our institution, acupuncture therapy is a consultation service requiring a clinician referral. Similarly, only 166 reasons for referral were documented in patients' charts during these treatments. Some of the acupuncture treatments had more than one referral source documented, as well as multiple reasons for referral. The most common reasons for acupuncture referrals were for management of pain (71 or $42.8 \%$ ) or nausea and vomiting (64 or $38.6 \%$ ). Other reasons for referrals included emergence delirium, mood, neurological or gastroenterological complaints, and issues with medication dependence or weaning (Table 2).

\section{Chief complaints treated}

In addition to the main reasons for referral, several other chief complaints were reported by patients and thus treated by the acupuncture provider during their acupuncture appointments. A total of 761 documented chief complaints were treated during the 454 acupuncture treatments with recorded data available for review. Pain indications and nausea and vomiting compromised over half of all chief complaints treated, with 354 (46.5\%) being chief complaints of pain and 149 (19.6\%) of nausea and vomiting. Other chief complaints treated included neuropathies or neurological dysfunction, fatigue,

\begin{tabular}{|l|c|c|}
\hline & $\mathbf{n}$ & $\%$ \\
\hline Pain & 71 & 42.8 \\
\hline Nausea/Vomiting & 64 & 38.6 \\
\hline Emergence Delirium & 13 & 7.8 \\
\hline Emotional/Mood Complaints & 5 & 3 \\
\hline Neuropathies/Neurological Dysfunction & 3 & 1.8 \\
\hline Diarrhea/Constipation & 2 & 1.2 \\
\hline Pain Medication Dependence/Weaning & 2 & 1.2 \\
\hline Immune Support & 1 & 0.6 \\
\hline Vertigo & 1 & 0.6 \\
\hline Shortness of Breath & 1 & 0.6 \\
\hline Fever & 1 & 0.6 \\
\hline Fatigue & 1 & 0.6 \\
\hline Insomnia/Sleep Disturbance & 1 & 0.6 \\
\hline
\end{tabular}

Table 2: Documented reasons for acupuncture referrals. 
Citation: Ralston-Wilson J, Tseng A, Oberg E, Sasagawa M, Doorenbos AZ, et al. (2013) Utilization of Acupuncture Therapy among Pediatric Oncology Patients at a Tertiary Care Pediatric Hospital. Altern Integ Med 2: 129. doi:10.4172/2327-5162.1000129

\begin{tabular}{|l|c|c|}
\hline & $\mathbf{n}$ & $\%$ \\
\hline Pain & 354 & 46.5 \\
\hline Nausea/Vomiting & 149 & 19.6 \\
\hline Neuropathies/Neurological Dysfunction & 76 & 10 \\
\hline Fatigue & 45 & 5.9 \\
\hline Insomnia/Sleep Disturbance & 42 & 5.5 \\
\hline Mouth/Gum/Throat Problems & 24 & 3.2 \\
\hline Emergence Delirium & 15 & 2 \\
\hline Diarrhea/Constipation & 15 & 2 \\
\hline Emotional/Mood Complaints & 9 & 1.2 \\
\hline General Discomfort & 9 & 1.2 \\
\hline Pain Medication Dependence/Weaning & 5 & 0.7 \\
\hline Dizziness/Vertigo & 3 & 0.4 \\
\hline Skin Changes & 2 & 0.3 \\
\hline Immune Support & 1 & 0.1 \\
\hline Other & 12 & 1.6 \\
\hline
\end{tabular}

Table 3: Chief complaints treated.

\begin{tabular}{|l|c|c|c|c|}
\hline & \multicolumn{3}{|c|}{ Yes } & No \\
\hline & $\mathbf{n}$ & $\%$ & $\mathbf{n}$ & $\%$ \\
\hline Tongue Diagnosis & 231 & 51 & 222 & 49 \\
\hline Pulse Diagnosis & 328 & 72.4 & 125 & 27.6 \\
\hline Abdominal Diagnosis & 139 & 30.7 & 314 & 69.3 \\
\hline TCM Diagnosis & 24 & 5.3 & 429 & 94.7 \\
\hline
\end{tabular}

sleep disturbance, oral cavity problems, emergence delirium, mood complaints, general discomfort, and pain medication dependence or weaning issues. Included in neuropathies/neurologic dysfunction were paralysis, ataxia, aphasia, and restless legs. Documentation of chief complaints was not available for 69 (13.2\%) treatments (Table 3).

\section{Additional treatment specific data}

Various diagnostic tools were employed by the acupuncture providers to determine an acupuncture diagnosis and appropriate acupuncture treatment plan to address the patient's complaints. Pulse diagnosis as a diagnostic tool, was most frequently (328 or $72.4 \%$ ) employed followed by tongue diagnosis (231 or $51 \%$ ) and abdominal diagnosis (139 or $30.7 \%$ ). A TCM diagnosis was infrequently documented ( 24 or $5.3 \%$ ) during these visits (Table 4 ).

While there was a wide range of acupuncture points used during treatments, there were a number of points that were most commonly used (Figure 3). The acupuncture points most frequently encountered in this analysis were ST-36 (Zusanli), LI-4 (Hegu), KD-3 (Taixi), SP-6 (Sanyinjiao), LV-3 (Taichong), PC-6 (Neiguan), DU-20 (Baihui), LI11 (Quchi), SJ-5 (Waiguan), and UB-60 (Kunlun). The top five most common acupuncture points used for management of pain or nausea and vomiting varied by only 1 point. While both groups included ST36 , LI-4, KD-3, and SP-6 as the common treatment acupoints, PC-6 was uniquely included in the acupuncture treatment of nausea and vomiting group, and LV-3 was uniquely included in the acupuncture treatment of pain group. Interestingly, of 149 treatments provided for the chief complaint of nausea/vomiting, 88 (59.1\%) of those treatments were also for a chief complaint of pain. Acupuncture point data was not available for 102 or $19.5 \%$ of treatments.

The stimulation of acupuncture points for treatment purpose was achieved by manual needle stimulation (312 or $70.9 \%$ ), press needle placement (144 or $32.7 \%$ ), magnet placement (179 or $40.7 \%$ ), moxibustion (11 or $2.5 \%$ ), electrical stimulation or electrostimulation of needles ( 23 or $5.2 \%$ ), cupping (72 or $16.4 \%$ ), acupressure (7 or $1.6 \%$ ), guasha (2 or $0.5 \%)$, and low intensity laser stimulation (13 or $3.0 \%$ ) (Figure 4). Modalities of acupuncture point stimulation were available for 440 acupuncture treatments available for review. Needles or press needles were used in over $75 \%$ of acupuncture treatments. When needles or press needles were used, a total needle count of 11-15 needles was most common, occurring in $36.7 \%$ of treatments. The duration of the acupuncture treatment when needle stimulation was used (needle retention time) was most commonly $16-20$ minutes as noted in $46.9 \%$ of treatments with documentation of this data.

\section{Acupuncture treatment response}

Pain score documentation by an acupuncture provider was available for $200(56.5 \%)$ treatments performed for a chief complaint of pain, of which only $9(2.5 \%)$ treatments had both pre- and post-treatment pain scores documented. Pain scores were significantly reduced after an acupuncture treatment among 8 of the nine treatments with preand post-acupuncture treatment pain scale documentation. The mean reduction in pain scores among these patients was 3.67.

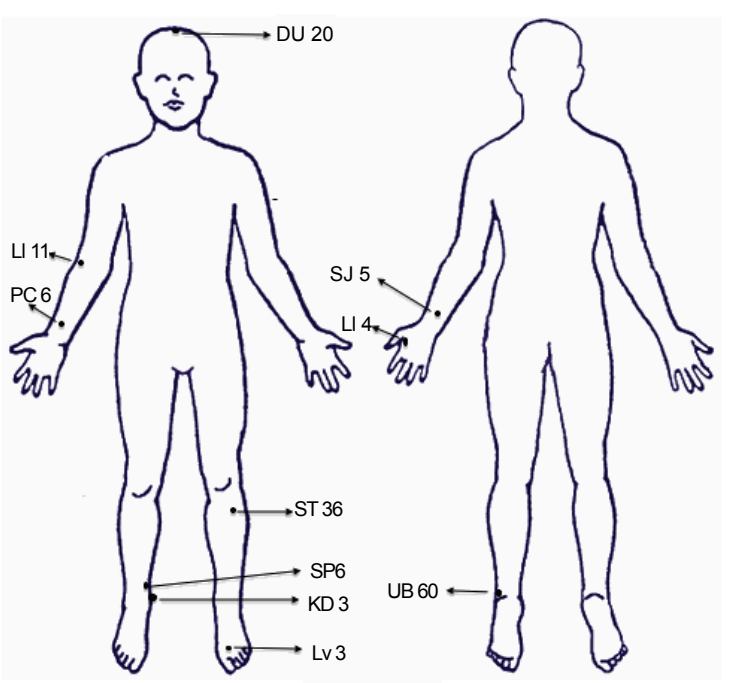

Figure 3: Most commonly used acupuncture points.

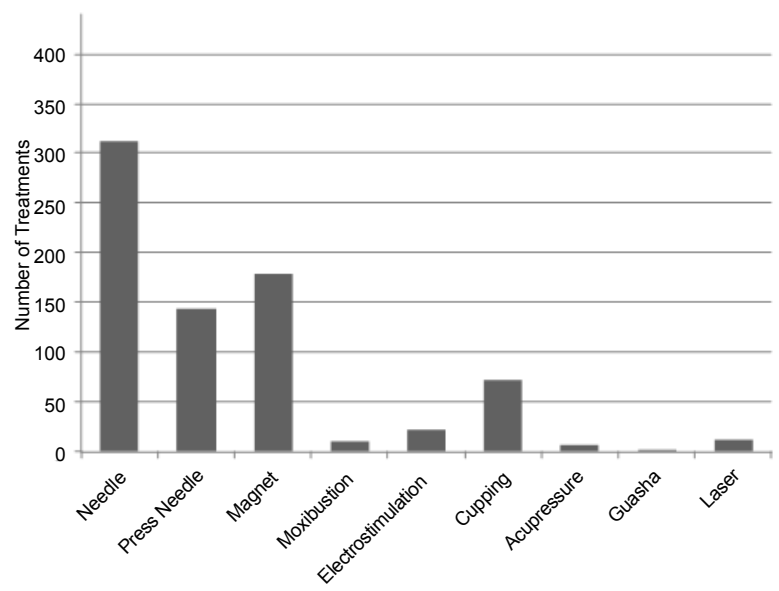

Figure 4: Acupuncture point stimulation. 


\begin{tabular}{|c|c|c|}
\hline & $\mathbf{n}$ & $\%$ \\
\hline \multicolumn{3}{|l|}{ Positive Response } \\
\hline Beneficial/Good response & 157 & 47.3 \\
\hline Improvement & 21 & 6.3 \\
\hline Relaxing/Able to nap & 16 & 4.8 \\
\hline Patient/Family requesting acupuncture & 8 & 2.4 \\
\hline Tolerated therapy well & 106 & 31.9 \\
\hline Open to having acupuncture & 1 & 0.3 \\
\hline \multicolumn{3}{|l|}{ Neutral Response } \\
\hline No change & 9 & 2.7 \\
\hline Denied offering of further treatment & 3 & 0.9 \\
\hline \multicolumn{3}{|l|}{ Negative Response } \\
\hline Worried/Unsure/Painful/Requesting less & 9 & 2.7 \\
\hline Felt worse (likely due to acupuncture) & 2 & 0.6 \\
\hline
\end{tabular}

Table 5: Patient/Family/Provider reported responses.

\begin{tabular}{|l|c|c|c|c|}
\hline & \multicolumn{2}{|c|}{ Current } & \multicolumn{2}{c|}{ Past } \\
\hline & $\mathbf{n}$ & $\%$ & $\mathbf{n}$ & $\%$ \\
\hline Allergic Reaction & 0 & 0 & 0 & 0 \\
\hline Bleeding & 0 & 0 & 0 & 0 \\
\hline Bruising or Hematoma & 0 & 0 & 0 & 0 \\
\hline Burn & 0 & 0 & 0 & 0 \\
\hline Discomfort & 12 & 1.5 & 0 & 0 \\
\hline Dizziness & 1 & 0.1 & 0 & 0 \\
\hline Fainting & 0 & 0 & 0 & 0 \\
\hline Fatigue & 0 & 0 & 0 & 0 \\
\hline Headache & 0 & 0 & 1 & 0.1 \\
\hline Infection & 0 & 0 & 0 & 0 \\
\hline Localized Skin Irritation & 1 & 0.1 & 4 & 0.5 \\
\hline Nerve Injury or Damage & 0 & 0 & 0 & 0 \\
\hline Pain at Puncture Site & 14 & 1.8 & 1 & 0.1 \\
\hline Pneumothorax & 0 & 0 & 0 & 0 \\
\hline Other Organ Puncture or Damage & 0 & 0 & 0 & 0 \\
\hline Spine or Spinal Cord Injury & 0 & 0 & 0 & 0 \\
\hline Other (please specify) & 0 & 0 & 0 & 0 \\
\hline None Mentioned & 426 & 93.8 & 315 & 98.1 \\
\hline
\end{tabular}

Table 6: Documented acupuncture-related adverse events.

Additionally, content analysis was performed to describe summary characteristics of patient/family/provider reported responses (Table 5). Out of 518 acupuncture treatments that were available for review, 269 $(51.9 \%)$ of these treatments had documented responses, while no data was available for the other 249 (48.1\%) treatments. Data was considered not available if no response to treatment was documented or the documentation was interpreted to be irrelevant to this information. Of the available data for these treatments, 332 responses were documented and considered significant for review. Three hundred and nine $(93.1 \%)$ treatment responses were interpreted to be positive, 12 (3.6\%) neutral, and $11(3.3 \%)$ negative.

Positive responses were determined based on interpretation of statements from patients, family, and providers. Comments such as "felt treatments were beneficial", "were feeling better", "noticed improvement attributable to acupuncture", "felt relaxed", or "experienced improvement in symptoms". Requests for further acupuncture treatments or tolerating the acupuncture treatments well were also considered consistent with a positive response. A neutral response was based on interpretation of statements that no change was documented or the patient denied the offering of further acupuncture treatment without mention of feeling better or worse due to acupuncture therapy. A negative response was determined based on interpretation of statements of patients feeling worried or unsure about acupuncture, expressing that treatments were painful, requesting less acupuncture therapy, or feeling worse when it was likely due to acupuncture treatments. If there were multiple statements reflecting positive or negative responses, they were considered a separate response, e.g. if a patient reported that their pain improved due to acupuncture and the practitioner or patient also reported that they were able to nap, 2 positive responses were recorded for this treatment.

\section{Acupuncture-related adverse events}

Thirty-four total adverse events were documented during 454 acupuncture treatments with documented data relevant to this review (Table 6). A total of 775 opportunities were available to document either current or past adverse events occurring attributable to acupuncture. There was a $4.4 \%$ incidence of adverse events documented based on our criteria, but none of these were a serious adverse event. All 34 reported adverse events were transient and mild including discomfort, dizziness, headache, skin irritation, and pain at the needling site. The majority of these ( 27 or $79.4 \%$ ) resolved at the time of needle withdrawal or by the conclusion of the treatment. If discomfort was noted, it was reported to be tolerable by the patient during the acupuncture treatment. Adverse event data was not available for 69 (13.2\%) treatments.

\section{Discussion}

While the cure rates for childhood cancers continue to rise with therapeutic success rates achievable in approximately $75 \%$ of all cancers occurring in childhood $[16,20]$, cancer treatment-related side effects may present unique challenges in the pediatric and young adult patient. Side effects that may occur due to conventional cancer treatments include fatigue, bone marrow suppression, nausea and vomiting, anorexia, mucositis, hair loss, cardiotoxicity, and/or liver and kidney damage $[9,21]$. Cancer treatment-related side effects may be mild and resolve at the completion of conventional therapy, while others may be serious and even life threatening [21-23]. Some chemotherapyinduced side effects may require a reduction in dosage or a delay in the subsequent cycle of chemotherapy [21,24,25].

Rate of utilization of complementary and alternative medicine (CAM) therapies among adult and pediatric oncology patients is reported to range from $7-91 \%[5,8,26,27]$ and $31-84 \%$ respectively $[13,16,18,28-31]$. Acupuncture therapy usage was not often singled out among the pediatric oncology population in these studies. CAM utilization among the general oncology population has been increasing [5]. Some of the adult cancer studies looking at CAM use have further examined the rates of acupuncture therapy utilization with reported rates to be as low as $1.7 \%[5,26]$ and up to $31 \%[5,32]$. One of the studies among adult cancer patients in Washington State reported acupuncture therapy usage to be $1.7 \%$ in the year 2000 [26]. The authors are not aware of any pediatric oncology studies specifically evaluating acupuncture therapy utilization among this oncology population. The results of our study indicate a relatively low incidence (3.2\%) of acupuncture therapy use among the pediatric oncology patients in the same geographic location as the adult cancer patients (Washington State). Although rates of acupuncture therapy use in pediatric patients may vary $[13,15]$, the lower incidence in the oncological population may be attributable to concerns about risks of infection and bleeding among this susceptible population with neutropenia and thrombocytopenia associated with their disease process or therapeutic interventions.

In our study, pain and nausea/vomiting were the two most common reasons triggering a referral for acupuncture therapy, highlighting the role these two symptoms play in the care of a cancer patient. These two 
symptoms constitute the top complaints with a high impact on the quality of life among cancer patients [1-9,13-15,33] thus, highlighting both the importance of management of these symptoms as well as inability to adequately treat these symptoms, in this population. Pharmacological agents used as therapeutic options for these symptoms may further result in more adverse effects [5,15,33-35]. Acupuncture as an adjuvant therapy, can aid in controlling these symptoms, improving the quality of life and reducing the incidence of dose-related side effects of pharmacological agents $[1-9,11,14,15,33]$. In our results, it was also notable that although pain and nausea/vomiting remained the most commonly reported and treated complaints, there were several other complaints that were identified and treated by the acupuncturists, either as additional complaints or in a higher incidence than at the time of referral (fatigue, insomnia, neuropathies).

The referral sources indicate that clinical providers were responsible for about $60 \%$ of acupuncture referrals, while $40 \%$ of acupuncture referrals were patient or family driven. We are not aware of any studies in the pediatric oncology population that characterize the patterns of referral for acupuncture therapy. Our study demonstrates that a majority ( $75 \%)$ of acupuncture treatments were performed by physician acupuncturists. This might in part be a reflection of the institutional culture and practice where a higher degree of comfort is allocated to an allopathic provider of CAM therapy and perhaps a slightly larger body of available evidence for acupuncture use in published CAM literature $[5,36]$.

Pulse and Tongue examinations were used most frequently as diagnostic tools for establishing an acupuncture diagnosis and acupuncture treatment plan. There can be a variation among acupuncture providers as to which diagnostic tools are felt pertinent in their clinical care. This variation may be attributable to the differences in their acupuncture training such as Chinese, Japanese, Korean, French Energetic acupuncture or medical acupuncture. Use of consistent diagnostic tools by different acupuncturists while performing acupuncture therapy could be instrumental in highlighting the fact that despite carrying the same cancer diagnoses, conventional therapy use, or chief complaints, patients may require different acupuncture treatment plans that are tailored to address their specific acupuncture diagnoses. Acupuncture treatment plans, not only addresses patients' chief complaints, but also take into consideration the constitutional differences and uniqueness among patients. Our study did not explore the relationship between acupuncture provider training and the type and frequency of TCM diagnostic tools used, nor are we aware of other studies investigating the use of TCM diagnostic tools in the hospital setting among pediatric oncology patients. However, such an investigation in the future may contribute valuable information regarding outcomes of acupuncture therapy based on individualized versus protocol-based treatment plans.

The data on safety and efficacy of acupuncture in the pediatric population for non-cancer related conditions [13-15,37] as well as that in the adult cancer population [4-6,8,38], suggests that when performed by qualified individuals, the incidence of adverse events is small. However, acupuncture research addressing these issues in the pediatric oncology population is very limited [12-15]. An overall rate of acupuncture-related adverse events of $4.4 \%$ among children with cancer diagnoses, is consistent with that reported in the prior two small studies.

The interventional study for chemotherapy-induced nausea and vomiting by Gottschling et al. [33] reported no adverse events; however pain from needling was reported in 4 out of 23 (17.4\%) patients, compared to our patients where this rate was only reported $1.8 \%$ of the time. In terms of bleeding complications, our patients did not experience any significant complications during acupuncture treatments, similar to the findings reported by Ladas et al. [12] in their retrospective review among pediatric cancer patients where at least $47 \%$ of them had thrombocytopenia at the time of acupuncture treatments. We did not specifically examine the blood counts of our patients at the time of acupuncture treatments in this review. Lower rates of adverse events in our study may be a result of under-reporting of adverse events based on incomplete data or a consequence of all acupuncture treatments being performed by well-trained and qualified acupuncture providers with specific training, experience, and knowledge of the pediatric and oncology population. While serious acupuncturerelated adverse events have been documented in literature, we did not encounter any serious or life threatening adverse events during our study. Although acupuncture is generally considered to be a safe modality when practiced by well-qualified and trained practitioners, special considerations in safety are warranted when considering this modality for both the pediatric and oncology population. Further prospective investigations assessing safety and efficacy in the pediatric oncology population are warranted to bolster evidence-based practice of acupuncture therapy in the management of cancer treatment-related side effects and improvements in quality of life.

Our study has some limitations including its retrospective design, lack of controls or randomization. Further, due to varying practices in documentation during the initial years of introduction of the acupuncture service, there was limited or incomplete data available for review, possibly affecting the results of our study. Furthermore, content analysis of patient responses to acupuncture therapy might be overreported for positive responses by the patients since they were collected and reported by the acupuncture provider and subsequent subjective interpretation of the documented responses by the reviewer.

\section{Conclusion}

In summary, acupuncture therapy use among the pediatric oncology population at our institution is well tolerated, sought both by clinical providers as well as the patients and their families, and safe when performed by providers with specific training and expertise in the pediatric and oncology population. In our patients, acupuncture therapy also proved to be a valuable adjuvant modality, not only for the pain and nausea/vomiting complaints, but also for supportive care through conventional treatments by addressing several other problems such as neuropathies/neurological dysfunction, fatigue, mood issues and sleep disturbance. Future research with prospective, randomized-controlled trials is warranted to evaluate safety, efficacy and acceptability of acupuncture therapy in this unique population that has a higher likelihood of complications and a higher incidence of disease and treatment-related side effects impacting their quality of life.

\section{Acknowledgement}

This project was supported by institutional funds from Bastyr University under award number BUCSR-Y4-001, Seattle Children's Hospital and by the National Cancer Institute of the National Institutes of Health under award number R42CA141875. The content is solely the responsibility of the authors and does not necessarily represent the official views of the National Institutes of Health or the individual institutions.

\section{References}

1. Lin JG, Chen YH (2012) The role of acupuncture in cancer supportive care. Am J Chin Med 40: 219-229.

2. Smith ME, Bauer-Wu S (2012) Traditional Chinese Medicine for cancer-related symptoms. Semin Oncol Nurs 28: 64-74. 
Citation: Ralston-Wilson J, Tseng A, Oberg E, Sasagawa M, Doorenbos AZ, et al. (2013) Utilization of Acupuncture Therapy among Pediatric Oncology Patients at a Tertiary Care Pediatric Hospital. Altern Integ Med 2: 129. doi:10.4172/2327-5162.1000129

3. O'Regan D, Filshie J (2010) Acupuncture and cancer. Auton Neurosci 157: 96100

4. Lu W, Rosenthal DS (2010) Recent advances in oncology acupuncture and safety considerations in practice. Curr Treat Options Oncol 11: 141-146.

5. Lu W, Dean-Clower E, Doherty-Gilman A, Rosenthal DS (2008) The value of acupuncture in cancer care. Hematol Oncol Clin North Am 22: 631-648, viii.

6. Deng G, Vickers A, Simon Yeung K, Cassileth BR (2006) Acupuncture: integration into cancer care. J Soc Integr Oncol 4: 86-92.

7. Lu W (2005) Acupuncture for side effects of chemoradiation therapy in cancer patients. Semin Oncol Nurs 21: 190-195.

8. Cohen AJ, Menter A, Hale L (2005) Acupuncture: role in comprehensive cancer care--a primer for the oncologist and review of the literature. Integr Cancer Ther 4: 131-143.

9. Conklin KA (2002) Acupuncture and the cancer patient. Medical Acupuncture 2: 23-31.

10. Johnstone PA, Niemtzow RC, Riffenburgh RH (2002) Acupuncture for xerostomia: clinical update. Cancer 94: 1151-1156.

11. Dundee JW, Ghaly RG, Fitzpatrick KT, Abram WP, Lynch GA (1989) Acupuncture prophylaxis of cancer chemotherapy-induced sickness. J R Soc Med 82: 268-271.

12. Ladas EJ, Rooney D, Taromina K, Ndao DH, Kelly KM (2010) The safety of acupuncture in children and adolescents with cancer therapy-related thrombocytopenia. Support Care Cancer 18: 1487-1490.

13. Gold JI, Nicolaou CD, Belmont KA, Katz AR, Benaron DM, et al. (2009) Pediatric acupuncture: a review of clinical research. Evid Based Complement Alternat Med 6: 429-439.

14. Jindal V, Ge A, Mansky PJ (2008) Safety and efficacy of acupuncture in children: a review of the evidence. J Pediatr Hematol Oncol 30: 431-442.

15. Kundu A, Berman B (2007) Acupuncture for pediatric pain and symptom management. Pediatr Clin North Am 54: 885-889.

16. Sencer SF, Kelly KM (2006) Bringing evidence to complementary and alternative medicine for children with cancer. J Pediatr Hematol Oncol 28: 186-189.

17. Kemper KJ, Sarah R, Silver-Highfield E, Xiarhos E, Barnes L, et al. (2000) On pins and needles? Pediatric pain patients' experience with acupuncture. Pediatrics 105: 941-947.

18. Ernst E (1999) Prevalence of complementary/alternative medicine for children: a systematic review. Eur J Pediatr 158: 7-11.

19. http://www.raosoft.com/samplesize.html. Raosoft ${ }^{\circledR}$ sample size calculator Access date: October 29, 2012

20. Ries LAG, Smith MA, Gurney JG, Linet M, Tamra T, et al. (1999) Cance incidence and survival among children and adolescents: United States SEER Program 1975-1995. National Cancer Institute (NIH), Bethesda, Maryland.

21. Abeloff MD, Armitage JO, Niederhuber JE, Kastan MB, McKenna WG (2008) Clinical Oncology. Churchill Livingstone Elsevier, Philadelphia, PA

22. Dobrasz G, Hatfield M, Jones LM, Berdis JJ, Miller EE, et al. (2013) Nursedriven protocols for febrile pediatric oncology patients. J Emerg Nurs 39: 289 295.

23. O'Brien SN, Blijlevens NM, Mahfouz TH, Anaissie EJ (2003) Infections in patients with hematological cancer: recent developments. Hematology Am Soc Hematol Educ Program .

24. Aapro M, Crawford J, Kamioner D (2010) Prophylaxis of chemotherapy-induced febrile neutropenia with granulocyte colony-stimulating factors: where are we now? Support Care Cancer 18: 529-541.

25. Foote M (1998) The Importance of Planned Dose of Chemotherapy on Time: Do We Need to Change Our Clinical Practice? Oncologist 3: 365-368.
26. Lafferty WE, Bellas A, Corage Baden A, Tyree PT, Standish LJ, et al. (2004) The use of complementary and alternative medical providers by insured cancer patients in Washington State. Cancer 100: 1522-1530.

27. Ernst E, Cassileth BR (1998) The prevalence of complementary/alternative medicine in cancer: a systematic review. Cancer 83: 777-782.

28. Kelly KM (2009) Integrative therapies for children with hematological malignancies. Hematology Am Soc Hematol Educ Program .

29. Neuhouser ML, Patterson RE, Schwartz SM, Hedderson MM, Bowen DJ, et al. (2001) Use of alternative medicine by children with cancer in Washington state. Prev Med 33: 347-354.

30. Whitsett SF, Anderson R, Coppes MJ (1999) Why are children with cance being exposed to complementary medicine? West J Med 171: 150-151.

31. Lee AC, Highfield ES, Berde CB, Kemper KJ (1999) Survey of acupuncturists: practice characteristics and pediatric care. West J Med 171: 153-157.

32. Morris KT, Johnson N, Homer L, Walts D (2000) A comparison of complementary therapy use between breast cancer patients and patients with other primary tumor sites. Am J Surg 179: 407-411.

33. Gottschling S, Reindl TK, Meyer S, Berrang J, Henze G, et al. (2008) Acupuncture to alleviate chemotherapy-induced nausea and vomiting in pediatric oncology - a randomized multicenter crossover pilot trial. Klin Padiatr 220: 365-370.

34. Villars P, Dodd M, West C, Koetters T, Paul SM, et al. (2007) Differences in the prevalence and severity of side effects based on type of analgesic prescription in patients with chronic cancer pain. J Pain Symptom Manage 33: 67-77.

35. Holdsworth MT, Raisch DW, Frost J (2006) Acute and delayed nausea and emesis control in pediatric oncology patients. Cancer 106: 931-940.

36. Kundu A, Tassone RF, Jimenez N, Seidel K, Valentine JK, et al. (2011) Attitudes, patterns of recommendation, and communication of pediatric providers about complementary and alternative medicine in a large metropolitan children's hospital. Clin Pediatr (Phila) 50: 153-158.

37. Adams D, Cheng F, Jou H, Aung S, Yasui Y, et al. (2011) The safety of pediatric acupuncture: a systematic review. Pediatrics 128: e1575-e1587.

38. MacPherson H, Thomas K, Walters S, Fitter M (2001) A prospective survey of adverse events and treatment reactions following 34,000 consultations with professional acupuncturists. Acupunct Med 19: 93-102. 